\title{
A Retrospective Study on Chronic Pelvic Pain Treated with Traditional Chinese Medicine Combined Therapy: 179 Cases (2016-2018)
}

\author{
Shanshan Guo \\ Yijia Chen \\ Qianjue Tang \\ Lian Liu \\ Lianwei $\mathrm{Xu}$ \\ Gynecology Department, Longhua \\ Hospital, Shanghai University of \\ Traditional Chinese Medicine, Shanghai, \\ 200032, People's Republic of China
}

Purpose: This study aims to evaluate clinical efficiency and side effects of combined therapy of traditional Chinese medicine in female treating chronic pelvic pain.

Methods: A retrospective study was conducted by analyzing 179 patients with chronic pelvic pain. They were divided into the 3-course group and the 1-course group. Patients in the 3-course group received combined therapy of traditional Chinese medicine 10 days per menstrual cycle for three months, while the 1-course group received the same combined therapy for only one menstrual cycle. VAS scores of five kinds of pain were compared between the two groups. Meanwhile, SF-36 scores, menstruation, leucorrhea and major accompanying symptoms were analyzed. To assess side effects of the combined therapy, safety indices were recorded before and after treatment as well.

Results: VAS scores of patients in the 3-course group after treatment were significantly lower than that before the treatment. Total scores of SF-36 were significantly improved after the treatment, and persistently improved even after the discontinuation of the treatment. The menstrual and leucorrhea condition improved as well after the treatment. In addition, among the concomitant symptoms, insomnia, loss of appetite, mental fatigue symptoms were significantly improved after the treatment. More importantly, significances were observed in the improvement of most VAS scores between patients who underwent a 1-course treatment and patients with a 3-course treatment. Meanwhile, no significant change was observed in safety indices before and after treatment. Possible adverse events related to the treatment plan for all enrolled patients included mild skin rash in 16 patients, stomachache in 3 patients after taking medicine on an empty stomach, and oral ulcer in 1 patient.

Conclusion: Combined therapy of traditional Chinese medicine alleviated symptoms of chronic pelvic pain, soothed the accompanying symptoms, and hence helped to improve the life quality of women.

Keywords: combined therapy of traditional Chinese medicine, chronic pain in pelvic area, retrospective study, efficacy

\section{Introduction}

The American College of Obstetricians and Gynecologists and the ReVITALize data definitions initiative define chronic pelvic pain (CPP) as "pain symptoms perceived to originate from pelvic organs/structures typically lasting more than 6 months". It is often associated with suggestive symptoms of lower urinary tract, sexual, bowel, pelvic floor, myofascial, or gynecological dysfunction, and accompanied with negative cognitive, behavioral, sexual and emotional consequences. The 2019 EAU Guidelines on chronic
Correspondence: Lianwei Xu Gynecology Department, Longhua Hospital, Shanghai University of

Traditional Chinese Medicine, 725 South

Wanping Road, Shanghai, 200032,

People's Republic of China

Tel/Fax +86-2I-64385700

Email lianweixu_2800@I63.com 
pelvic pain indicate that etiological factors are complex, including pain caused by multiple systems such as reproductive system, urinary system, digestive system, and nervous system. Besides persistent lower abdominal pain, clinical manifestations also include dysmenorrhea, sexual intercourse pain, lumbosacral pain and anal drop pain. Yosef et al used baseline cross-sectional data from an ongoing prospective cohort to analyze and found that $55 \%$ of 656 women with chronic pelvic pain were diagnosed with endometriosis. ${ }^{1}$ Chronic pelvic inflammation disease and pelvic adhesion due to gynecologic surgery are also major factors to chronic pelvic pain. ${ }^{2}$ In addition to abdominal pain, infertility and pelvic lumps sometimes occur in a combined manner among gynecological outpatient patients.

The treatments of CPP varies according to the cause of the disease. Main treatments for pelvic pain caused by endometriosis include medication of hormones in general, and/or surgical treatments to resect lesions for endometriosis preferable for those with infertility, adnexal mass or ineffective medication. ${ }^{3}$ Meanwhile, oral and intravenous antibiotics are the main treatment for pelvic inflammation induced $\mathrm{CPP},{ }^{4}$ while pelvic adhesiolysis are the main treatment for severe pelvic (including intestinal) adhesions induced CPP. Certain adverse effects have been observed in the above-mentioned treatments: long-term oral uptake of hormones can cause gastrointestinal reactions, weight gain, and abnormal liver function; ${ }^{3}$ long-term use of antibiotics is prone to the resistance, microflora disorders, and damages of liver and kidney damage; ${ }^{5}$ surgical treatment can alleviate the abdominal pain of patients with endometriosis, but drug maintenance treatment is needed after operation to lower the recurrence rate and patients are usually afraid of surgery. Hence, the above three methods have to be compromised due to the low compliance of patients. Recently, a multidisciplinary treatment method, which consists of various approaches from physiatrists, pain physicians, obstetricians-gynecologists, urologists, gastroenterologists, primary care providers, physical therapists, psychiatrists and psychologists, has been suggested for the treatment of CPP. ${ }^{6}$ However, most of these studies focused on the treatment of interstitial cystitis/ painful bladder syndrome, while few aimed to alleviated pain from gynecological complexities.

Traditional Chinese medicine (TCM) has been used for a long time in the clinical practice of gynecology in Longhua Hospital to treat various symptoms of intractable pain caused by endometriosis and pelvic inflammation. According to the etiology and pathogenesis of CPP in TCM, such as dampness and turbidity, cold-heat mixture,
Qi stagnation, blood stasis, and collateral obstruction, comprehensive treatment was carried out by orally, intravenous infusion, external application, and retention enema for regulating Qi, clearing heat, warming meridians, removing dampness, removing blood stasis and relieve pain in the current study. Local therapies, such as external application and enema, can relieve pain by stimulating intestinal peristalsis, releasing intestinal adhesion, improving pelvic microcirculation blood flow changes, promoting vasodilatation, accelerating the absorption of inflammatory exudates and metabolism. Local hyperthermia can make drugs diffuse faster in the pelvic cavity, enhance drug absorption, and increase the therapeutic effect.

Astragalus membranaceus and Danhong injection were selected as the injection therapy for their compatibility. Astragalus membranaceus has the functions of invigorating Qi, stopping perspiration, diuresis, detumescence and purulent discharge. Salvia miltiorrhiza has the functions of activating blood circulation and removing blood stasis, nourishing blood and tranquilizing mind, cooling blood and detumescence. Safflower can promote blood circulation, dredge blood stasis and relieve pain. The two drugs complement each other and have the functions of activating blood circulation, removing blood stasis, and dredging channels and collaterals. Modern pharmacology showed that many effective ingredients in Astragalus, such as polysaccharide, flavonoids, saponins, amino acids and trace elements, could enhance immune function, promote the formation of antibodies in the body significantly, participate in the immune regulation of the body, and promote the production of immune-factors. Danhong injection can improve blood rheology and coagulation function, inhibit inflammation and oxidative stress reaction, repair and protect vascular injury, protect vascular endothelium, regulate vascular endothelial cell secretion and vasomotor function, and improve peripheral neuropathy. ${ }^{7}$ As a result, the combination of the two can improve the immune function of patients, improve blood flow in pelvic cavity, protect and repair blood vessels as well as peripheral nerves, and achieve the effect of relieving pelvic pain.

Combined therapy of TCM improved the quality of CPP patients' life and is well accepted by patients in our practice. However, to date, not detailed report on this topic had been published. Therefore, in this study, a retrospective study and analysis of the short-term and long-term efficiency of combined therapy of TCM on CPP has been investigated. 


\section{Methods}

\section{Design and Recruitment of Patients}

The current study was performed under the ethical instructions of the Longhua Hospital and reviewed by the committee. Furthermore, the study was conducted in accordance with the principles of the Declaration of Helsinki.

The retrospective study was done by analyzing 179 female patients diagnosed with chronic pelvic pain in Longhua Hospital from Jan. 2016 to Sept. 2018. The records of patients who met the inclusion criteria and had received combined therapy of traditional Chinese medicine for at least 10 days per month were selected in this study.

The inclusion criteria:

1. Clinically diagnosis of patients was in accordance with CCP;

2. Female patient's age ranged 20 to 60 ;

3. Informed consent to treatment.

The exclusion criteria included:

1. Chronic pelvic pain resulted from nongynecological diseases;

2. In complications with serious heart, liver, kidney, hematopoietic system, or other medical diseases;

3. During pregnancy or lactation;

4. Patients who have used analgesics or hormones in the past six months;

5. Patients with a history of drug allergy;

6. Unable to cooperate, such as those with psychiatric disorders.

\section{Pre-Treatment Procedure}

Informed consent had been filled by willing-to-participate patients in accordance with combined therapy of Traditional Chinese medicine by the management committee of Gynecology of Longhua Hospital Affiliated Shanghai University of Traditional Chinese Medicine. Records of safety indices including routine examinations of blood and urine, vaginal secretions examination (leucorrhea routine, Chlamydia, mycoplasma and Neisseria gonorrhoeae culture), test of liver and kidney function, and electrocardiogram were collected for all subjects.

Pre-treatment pain index assessment was recorded as visual analogue scale (VAS) score. Quality of life was evaluated by Study Short Form-36 (SF-36) scale score, while symptom improvement (including menstruation, leucorrhea, and other related symptoms) was assessed by CMSS (Cox Menstrual Symptom Scale) score.

\section{Treatment Procedure}

Patients who underwent combined therapy of traditional Chinese medicine was analyzed in this study, including a combination of oral Chinese medicine, intravenous infusion, external application, and retention enema. A whole treatment course was composed from the third day after the menstrual period, with a duration of 10 days per month. Sex life was forbidden during the treatment. Patients were asked to adjust their living habits, no overworking, no staying up late, relax the mood, and light diet, as the doctor's advice. Combined therapy of traditional Chinese medicine was used as follows:

1. Oral application: "Pen Qing Fang" (from Sichuan New-Green Pharmaceutical Technology Development Co., Ltd.) is a New-Green prescription granule composed of 14 herbs and has a practical history of more than 10 years in Shanghai. The drug composition is shown in Table 1 (Chen's gynecological therapy in Shanghai intangible cultural heritage). After soaking with $100 \mathrm{~mL}$ boiling water, the drug was taken after meals twice a day.

2. Intravenous infusion: Astragalus injection (from Zhengda Qingchunbao Pharmaceutical Co., Ltd.) $20 \mathrm{~mL}$, Danhong injection (Shandong Danhong Pharmaceutical Co., Ltd.) $30 \mathrm{~mL}$, and $5 \%$ glucose injection $50 \mathrm{~mL}$. After mixing with syringe, drip intravenously once a day.

3. External application: The Pharmacy Department of Longhua Hospital provides external application of a compound prescription consists of 12 herbs. The preparation of the prescription is as follows: After soaking for 30 minutes, the herbs are cooked by a Chinese medicine decocting machine (Equipment type YJD20-GL) for 30 minutes, and then concentrated. Application happened with a cotton pad soaking $100 \mathrm{~mL}$ of the juice, placing to the lower abdomen, and irradiating with infrared ray for 20 minutes. This treatment applied once a day and the drug composition is shown in Table 2 (Chen's gynecological therapy in Shanghai intangible cultural heritage). 
Table I Recipe for the Oral Chinese Medicine

\begin{tabular}{|l|l|l|l|}
\hline Chinese Term (Pingying) & English Term & Latin Term & Daily Dose (g) \\
\hline Chuan lian zi & Chinaberry fruit & Fructus Toosendan & 9 \\
Yan hu suo & Corydalis Yanhusuo & Corydalis Rhizoma & 15 \\
Sheng pu huang & Pollen cattail & Pollen Typhae & 18 \\
Shan zha & Hawthorn & Fructus Crataegi & 15 \\
Di long & Pberetima & Pberetima & 9 \\
Tao ren & Peach kernel & Semen Persicae & 12 \\
Huang qi & Astragalus membranaceus & Radix Astragali & 15 \\
Pu gong ying & Dandelion & Herba Taraxaci & 30 \\
Di ding cao & Tokyo violet herb & Herba Violae & 15 \\
Bai jiang cao & Patrinia & Herba Patriniae & 15 \\
Ji xue teng & Lignum millettiae & Caulis Sargentodoxae & 15 \\
Chi shao & Radix Paeoniae & Radix Paeoniae Rubra & 15 \\
Dan shen & The root of red-rooted salvia & Radix Salviae Miltiorrhizae & 15 \\
Dang gui & Angelica & Radix Angelicae Sinensis & 9 \\
\hline
\end{tabular}

Table 2 Recipe for the External Application of Chinese Medicine

\begin{tabular}{|c|c|c|c|}
\hline Chinese Term (Pingying) & English Term & Latin Term & Daily Dose (g) \\
\hline Dang gui & Angelica & Radix Angelicae Sinensis & 15 \\
\hline Chuan xiong & Ligusticum wallichii & Rhizoma Chuanxiong & 15 \\
\hline Chi shao & Radix Paeoniae & Radix Paeoniae Rubra & 15 \\
\hline Tou gu cao & Tuberculate Speranskia herb & Phryma leptostachya & 30 \\
\hline Wu tou & Monkshood & Radix Aconiti & 15 \\
\hline Ji xue teng & Lignum millettiae & Caulis Sargentodoxae & 15 \\
\hline Bai zhi & Radix Angelicae dahuricae & Radix Angelicae Dahuricae & 15 \\
\hline Zao jiao ci & Spina gleditsiae & Fructus Gleditsiae Abnormalis & 15 \\
\hline Qiang huo & Notopterygium root & Rhizoma Et Radix Notopterygii & 15 \\
\hline Du huo & Radix angelicae tuhuo & Radix Angelicae Pubescentis & 15 \\
\hline Qian nian jian & Rhizoma homalonemae & Rhizoma Homalomenae & 15 \\
\hline Wu jia pi & Acanthopanax & Cortex Acanthopanacis & 15 \\
\hline
\end{tabular}

4. Retention enema: The Pharmacy Department of Longhua Hospital provides retention enema of Chinese medicine consists of 11 herbs. The herbs should be soaked for 30 minutes, cooked by Chinese medicine decocting machine (Equipment type YJD20-GL) for 30 minutes, and concentrated into $100 \mathrm{~mL}$ of juice. The retention enema is applied once a day. The drug composition is shown in Table 3 (Chen's gynecological therapy in Shanghai intangible cultural heritage).

\section{Grouping of the Patients}

Patients included in the current study were divided into two groups according to their treatment: a 3-course group who received treatment for 3 menstrual cycles and a 1-course group who received treatment for only 1 menstrual cycle. All subjects were asked to fill in VAS scores at three time points: immediately after treatment, 6 months after treatment and 12 months after treatment. Patients with pregnancy were recorded. Safety indices were reviewed by all patients. The 3-course group was also asked to fill in SF-36 and CMSS scores during the treatment.

\section{Outcome Measurements}

1. The VAS score: ${ }^{8-10}$ It is a scale to assess abdominal pain symptoms, using a $10-\mathrm{cm}$ line divided into 10 equal sections, with 0 representing "no pain", 1-3 for mild pain, 4-6 for moderate pain, and $>6$ for 
Table 3 Recipe for the Retention Enema of Chinese Medicine

\begin{tabular}{|l|l|l|l|}
\hline Chinese Term (Pingying) & English Term & Latin Term & Daily Dose (g) \\
\hline Yi yi ren & Coix seed & Semen Coicis & 15 \\
Tao ren & Peach kernel & Semen Persicae & 9 \\
Lu lu tong & Herb-medicine & Fructus Liquidambaris & 15 \\
Zao jiao ci & Spina gleditsiae & Fructus Gleditsiae Abnormalis & 12 \\
Hai zao & Seaweed & Sargassum & 15 \\
Hong hua & Safflower & Flos Carthami & 9 \\
Ai ye & Artemisia & Folium Artemisiae Argyi & 9 \\
Bai zhi & Radix Angelicae dahuricae & Radix Angelicae Dahuricae & 9 \\
Yu jin & Tulip & Radix Curcmae & 9 \\
Gui zhi & Cinnamon branch & Ramulus Cinnamomi & 9 \\
Dan shen & The root of red-rooted salvia & Radix Salviae Miltiorrhizae & 15 \\
\hline
\end{tabular}

severe pain, 10 representing "unbearable pain". Each participant was asked to indicate the level of pain in their lower abdominal and pelvic region on the scale. If the patient had other pain, including dysmenorrheal, lumbosacral pain, dyspareunia, and anal drop pain, the same had been noted.

2. The SF-36 score: ${ }^{11}$ It is used to assess the quality of life, and the maximum score achieved was 100 . In addition to evaluating the total score of SF, total scores of PF (physiological function), RP (role-physical), BP (bodily pain), GH (general health), VT (vitality), SF (social function), RE (role-emotional), and $\mathrm{MH}$ (mental health) were also evaluated.

3. The CMSS score: ${ }^{12}$ It is a scale ranging from 0 to 3 representing none, mild, moderate and severe symptoms perceived, respectively. Menstruation, leucorrhea and other related symptoms were included.

3.1. Menstruation: The following information was included: menstrual color, texture, odor, cycle (normal, early or late within 1 week, 7-14 days, and $>2$ weeks), menstrual period (normal, menstrual time $>7$ days, $7-14$ days, and $>15$ days), menstrual flow (normal, increase or decrease by 1/3,1/2, and double), dysmenorrhea, and blood clots.

3.2 Leucorrhea: The following information was included: volume, color, texture, odor, whether or not itching. According to TCM scale score, each item was divided into $0,1,2$, and 3 representing none, light, medium, and heavy, respectively. Each item was added up to count the overall score.

3.3 Other related symptoms: The following information was included: anxiety, loss of libido, insomnia, poor appetite and fatigue. According to the score of TCM scale, each item was divided into $0,1,2$, and 3 representing no, light, medium, and heavy, respectively. The number of improvements for each symptom was counted based on the change in the score.

1. Pregnancy: information was recorded for those who get pregnant within 12 months after the treatment.

\section{Data Analysis}

Mean and standard deviation were calculated and used for the statistics. Improvements of scores of VAS, SF-36, leucorrhea, and other related symptoms in the 3-course group before and after the treatment at various time points were assessed using Kruskal-Wallis tests. One-way Analysis of covariance (ANOVA) was used for the comparison between menstruation improvement in the 3-course group and that in the 1-course group. Mann-Whitney tests were used for the VAS improvement between the 1-course group and the 3 -course group at various time points. Statistical analysis was performed using Statistical Package for the Social Sciences (SPSS) version 21. A probability level of 0.05 or less was considered to be statistically significant.

\section{Results}

The General Information of Patients

A total number of 199 patients were enrolled. Data from 172 patients were included in the analysis (the detailed procedure is presented in Figure 1). After identification, the baseline clinical characteristics for both groups were compared in terms of age, height, weight, BMI, score of VAS and SF36, menstruation and leucorrhea (as shown in Table 4). Survey data on treatment courses, causes, purposes, pain type and related symptoms are listed in Table 5. 


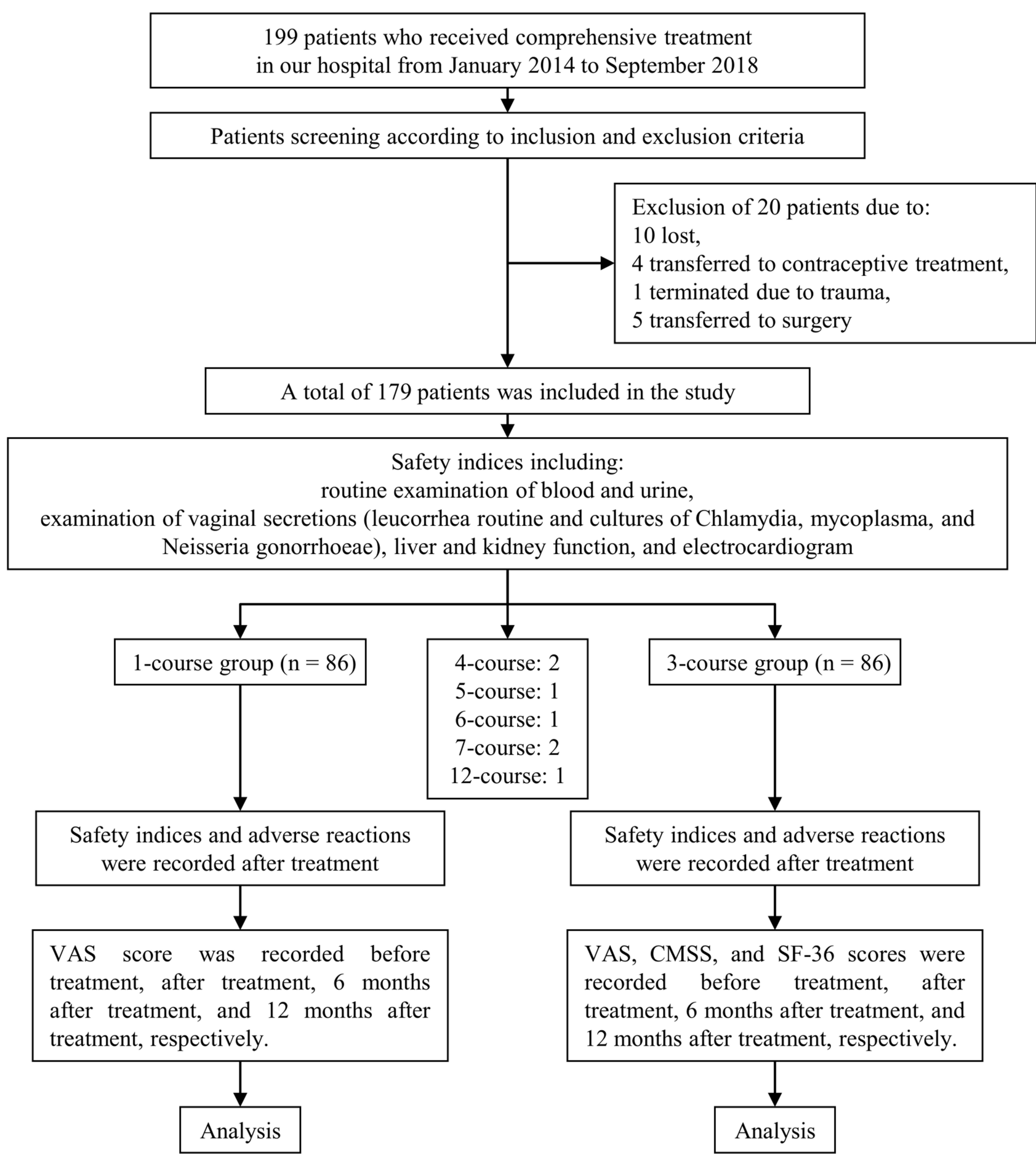

Figure I The detailed procedure of the current study.

\section{The Analysis of Curative Effects}

In the 3-course group, VAS and SF-36 (except BP) scores were significantly lowered after the treatment. Other symptoms such as menstrual, insomnia, poor appetite and fatigue were significantly improved $(p<0.05)$. Comparing with the baseline, BP (bodily pain) scores in SF-36 decreased significantly and other symptoms including leucorrhea, anxiety, and loss of libido were ameliorated 6 months after treatment and 12 months after treatment $(p<0.05)$ as shown in Figures 2-5.

In the 1-course group, the score of abdominal pain VAS tests after treatment was significantly lower than 
Table 4 Baseline of the Patients Enrolled

\begin{tabular}{|l|c|}
\hline Age (Years) & $\mathbf{3 5 . 9 7} \pm \mathbf{8 . 5 4 6}$ \\
\hline Height (meters) & $1.64 \pm 0.078$ \\
\hline Weight (kilograms) & $52.96 \pm 3.467$ \\
\hline BMI & $20.34 \pm 1.678$ \\
\hline VAS & \\
Abdominal pain & $5.86 \pm 2.171$ \\
Dysmenorrhea & $6.88 \pm 2.029$ \\
Dyspareunia & $6.81 \pm 1.678$ \\
Lumbosacral pain & $6.17 \pm 1.909$ \\
Anal drop pain & $6.56 \pm 1.875$ \\
\hline SF-36 & \\
Total score of SF-36 & $24.56 \pm 6.879$ \\
PF & $19.34 \pm 14.387$ \\
RP & $16.56 \pm 11.024$ \\
BP & $25.78 \pm 12.890$ \\
GH & $27.34 \pm 9.789$ \\
VT & $28.87 \pm 9.098$ \\
SF & $26.23 \pm 11.678$ \\
RE & $15.89 \pm 20.832$ \\
MH & $26.32 \pm 10.124$ \\
\hline Menstruation (days) & $6.64 \pm 4.437$ \\
\hline Leucorrhea (days) & $3.42 \pm 2.890$ \\
\hline
\end{tabular}

that at the baseline $(p<0.05)$. Figure 6 demonstrates that no significance was observed in the VAS score of dysmenorrheal, lumbosacral pain, dyspareunia, and anal drop pain, comparing with scores at the baseline to that of 12 months after treatment $(p>0.05)$.

No significance on scores was uncovered between the 1-course group and the 3-course group at baseline, while significant lower scores $(p<0.05)$ of all kinds of VAS tests were observed in the 3 -course group at all 3 time points after the treatment, comparing with that of the 1-course group.

Moreover, 9 pregnancies were recorded in the 3-course group while 5 in the 1-course group. 7 of them were not included in the statistics due to the exclusion criteria though the follow-up records showed that their VAS scores and clinical symptoms improved significantly.

\section{The Analysis of Safety Indices}

Adverse events recorded are listed in Table 6. There was no significant abnormality in safety indices after the treatment. Among them, 16 patients (8.94\% of total patients) had mild skin rash, 10 of them $(5.59 \%$ of total patients)
Table 5 Case Distribution

\begin{tabular}{|l|c|c|}
\hline & Cases & Percentage \\
\hline Causes & & \\
Acute pelvic inflammatory disease & 43 & $24.02 \%$ \\
Endometriosis & 55 & $30.73 \%$ \\
Operation & 61 & $34.08 \%$ \\
Unknown & 20 & $11.17 \%$ \\
\hline Purposes & & \\
Abdominal pain & 123 & $68.72 \%$ \\
Abdominal pain with infertility & 30 & $16.76 \%$ \\
Abdominal pain with mass & 26 & $14.53 \%$ \\
\hline Pain type & & \\
Dysmenorrhea & 42 & $23.46 \%$ \\
Dyspareunia & 36 & $20.11 \%$ \\
Lumbosacral pain & 53 & $29.61 \%$ \\
Anal drop pain & 39 & $21.79 \%$ \\
One kind of pain & 70 & $39.11 \%$ \\
Two kind of pain & 46 & $25.70 \%$ \\
Three kind of pain & 35 & $19.55 \%$ \\
Four kind of pain & 28 & $15.64 \%$ \\
\hline Related symptoms & 60 & $33.52 \%$ \\
Anxiety & 40 & $22.35 \%$ \\
Insomnia & 120 & $67.04 \%$ \\
Loss of appetite & 56 & $48.04 \%$ \\
Fatigue & & \\
Loss of libido & & \\
\hline
\end{tabular}

requested termination of treatment after one course of treatment, 2 (1.12\% of total patients) stopped after two courses, and $4(2.23 \%$ of total patients $)$ finished three courses. All patients with rashes recovered on their own.

1 patient $(0.56 \%$ of total patients) developed oral ulcer during the treatment. Considering the possibility of druginduced ulcer, the treatment was discontinued after one course of treatment.

4 patients (2.23\% of total patients) suffered from irregular bleeding during the treatment period. B-ultrasound results indicated endometrial thickening. It was found that they had a history of intermittent bleeding before the treatment and hence considered as irrelevant with the therapy.

Amenorrhea was recorded in 2 patients $(1.12 \%$ of total patients) aged 49 and 51, respectively. Review indicated their FSH levels were $23.56 \mathrm{mIU} / \mathrm{mL}$ and $46.52 \mathrm{mIU} / \mathrm{mL}$ respectively, suggesting the possible occurrence of menopause, and hence they were excluded from the therapy.

3 patients (1.68\% of total patients) had stomachache during the first course of treatment. Further inquiry 
A

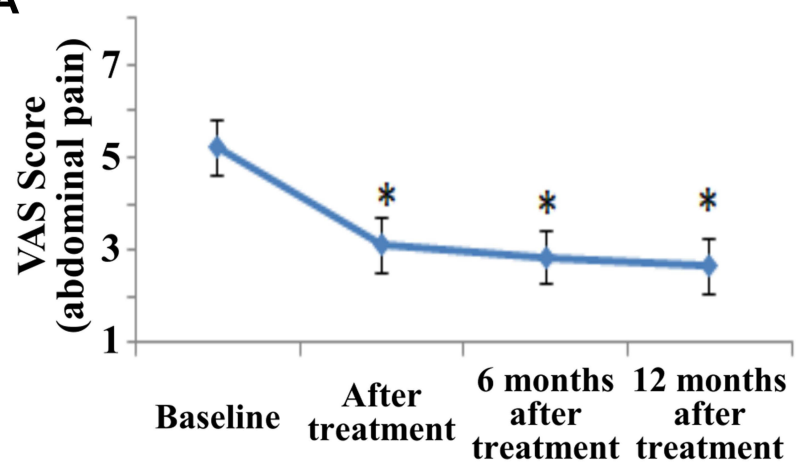

C
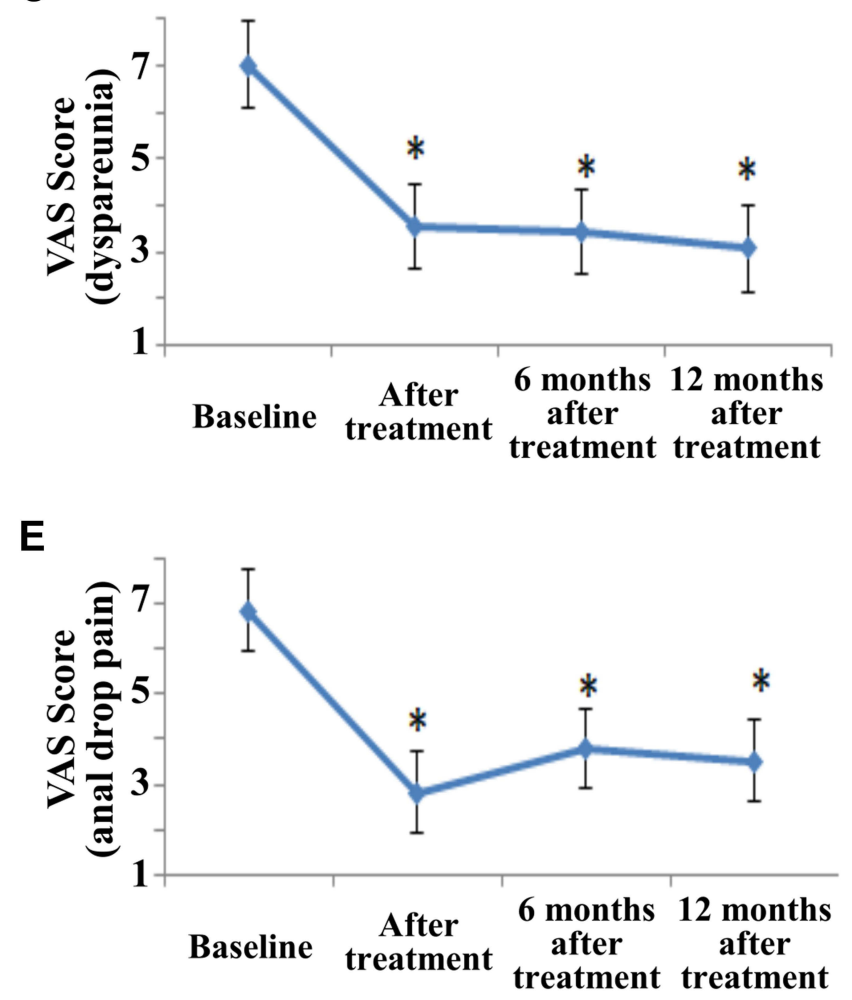

B

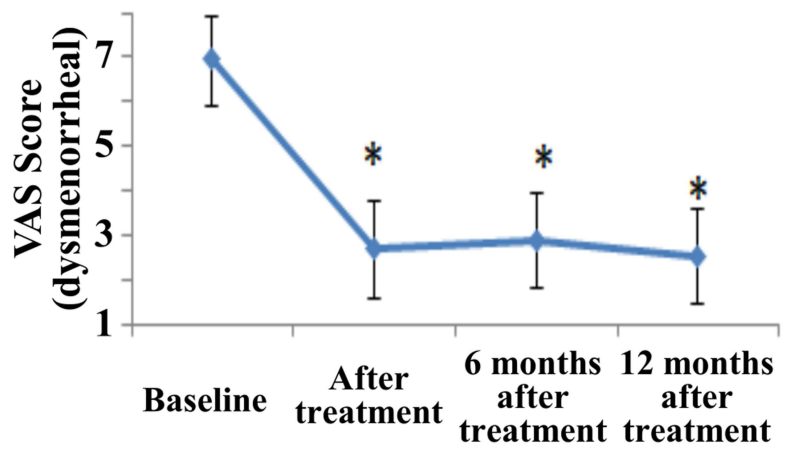

D

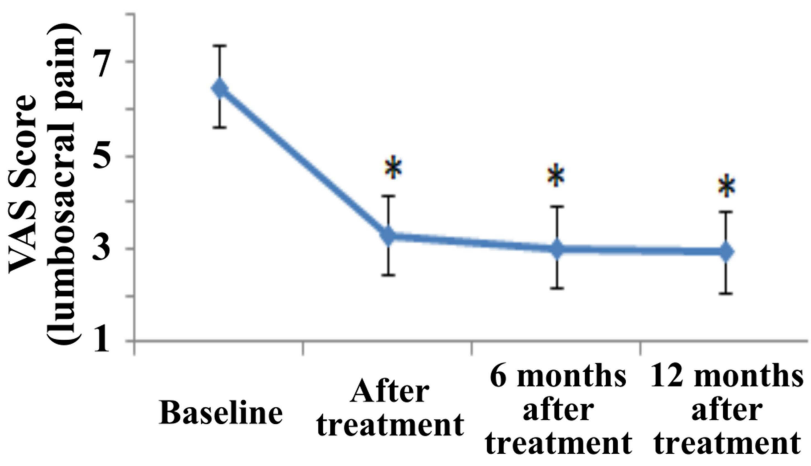

Figure 2 VAS scores in abdominal pain (A), dysmenorrheal (B), dyspareunia (C), lumbosacral pain (D), and anal drop pain (E) decreased significantly after the treatment in the 3-course group. *Indicated a significant difference with a $p<0.05$.

revealed that they took medicine on an empty stomach in the morning. After obeying the doctor's order of taking medicine after meals, their stomachache disappeared.

\section{Discussion}

Recent studies have shown that Chinese medicine could be an alternative therapy for CPP. Xuejuan used pelvic floor acupoint injection combined with warming meridians and dredging collaterals to treat 40 cases of pelvic pain of cold coagulation and blood stasis type with an effective rate of $85 \%{ }^{13}$ Yan-jun used oral Chinese medicine combined with enema to treat chronic pelvic pain patients, and the effective rate was $40 \% .{ }^{14}$ Chang-hui treated 36 patients with chronic pelvic pain by external application of traditional Chinese medicine, and the effective rate was $88.99 \% .{ }^{15}$ Our hospital has used traditional Chinese medicine for many years to treat CPP through various ways, but no detailed evaluation of the curative effect was conducted. Therefore, the main purpose of the current study was to determine the therapeutic efficacy of Combined therapy of traditional Chinese medicine in the management of CPP.

Patients treated for 3 months claimed significant improvement in pain perception before and after treatment, and the 
A

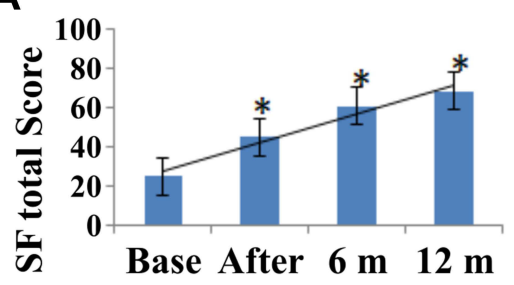

D

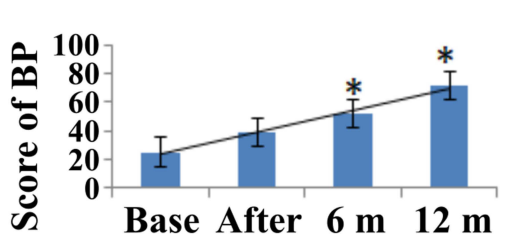

B

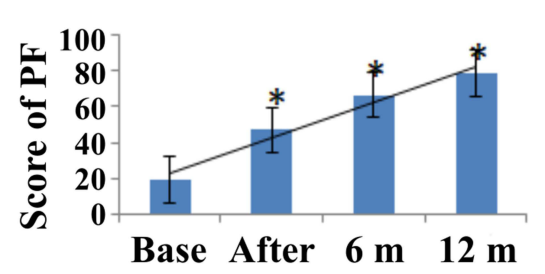

E

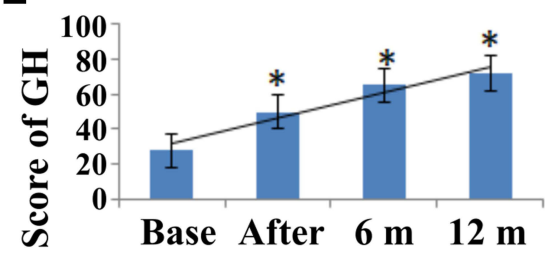

C

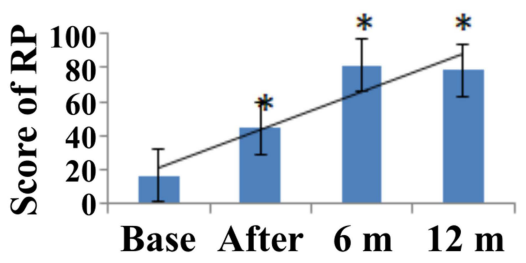

$\mathbf{F}$

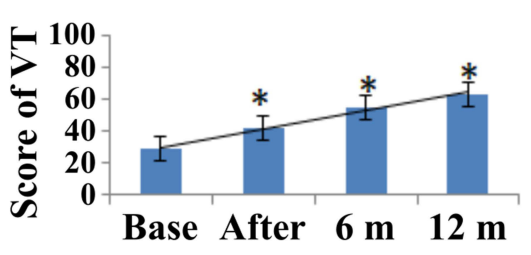

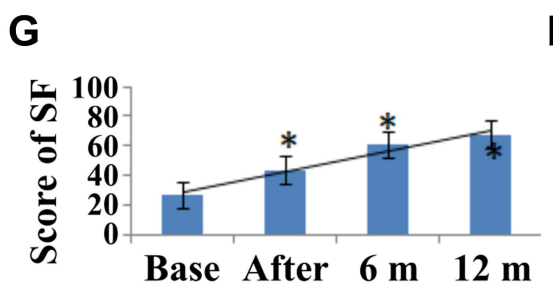
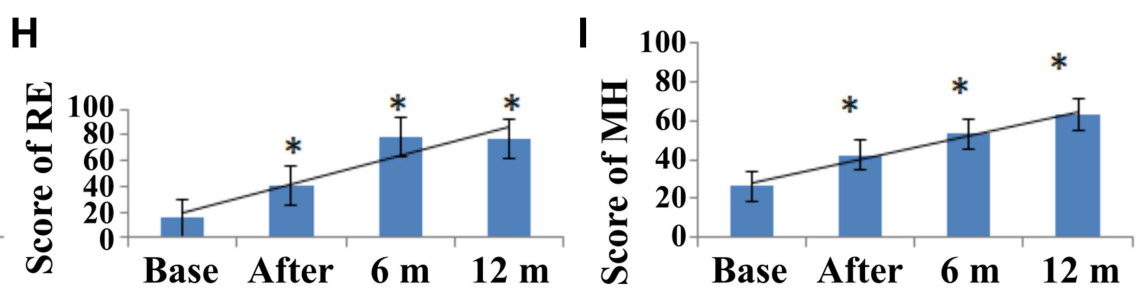

Figure 3 Scores of SF-36 in total score (A), PF (B), RP (C), BP (D), GH (E), VT (F), SF (G), RE (H), and MH (I) increased continuously after the treatment in the 3-course group. *Indicated a significant difference with a $p<0.05$. Base represents the baseline; After represents after treatment; $6 \mathrm{~m}$ represents 6 months after treatment; $12 \mathrm{~m}$ represents 12 months after treatment.

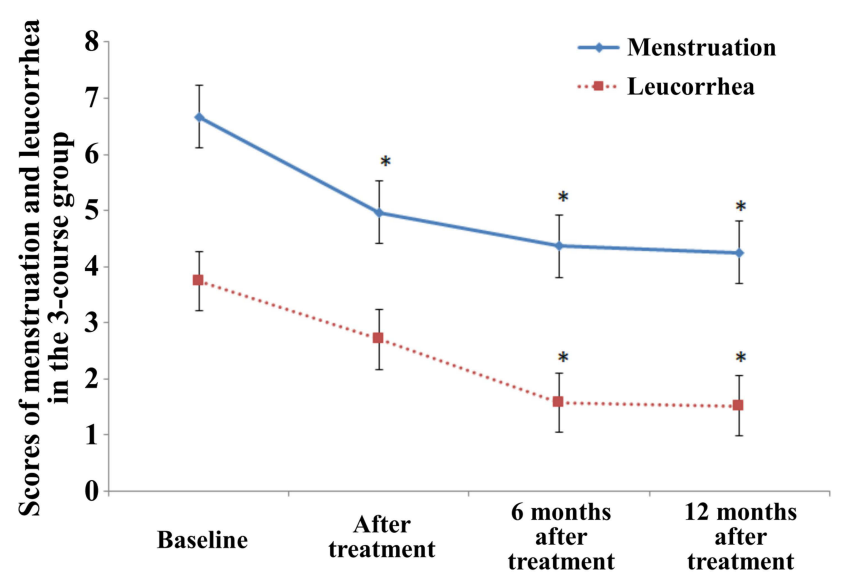

Figure 4 Scores of CMSS in menstruation and leucorrhea in the 3-course group. *Indicated a significant difference with a $p<0.05$.

alleviation remained significant at 1 year after the treatment, indicating that the 3-course of comprehensive treatment had a prolonged effect in pain soothing. Analysis on the SF-36 scores also implied the same trend, suggesting that apart from the pain, the quality of patients' life improved gradually as well. This means that the treatment as well as the doctor's guidance to patients on standardizing their life habits and diet would both contribute greatly to the relief of pain and the improvement of the quality of life.

In addition, menstrual disorders were significantly improved after the treatment, indicating that comprehensive treatment also has a prolonged effect in improving menstrual conditions. No significant difference in leucorrhea were observed after treatment, but significance showed in 6 months and 12 months. Considering a gradual improvement of patients' daily life, the leucorrhea situation was also getting better slowly.

The symptoms of insomnia, poor appetite and fatigue were alleviated after treatment, indicating that comprehensive treatment also has a certain effect on improving the above three symptoms. The symptoms of patients with anxiety and loss of libido were not relieved significantly after treatment, but these symptoms were significantly improved after 6 months and 1 year of observation after discontinuation of treatment. Based on the analysis of RE and MH scores in SF-36, it would be plausible to consider that anxiety and loss of libido are not only correlated by pelvic pain but also by patients' health and mental state. After treatment, although the abdominal pain was relieved, the discomfort in other aspects did not 


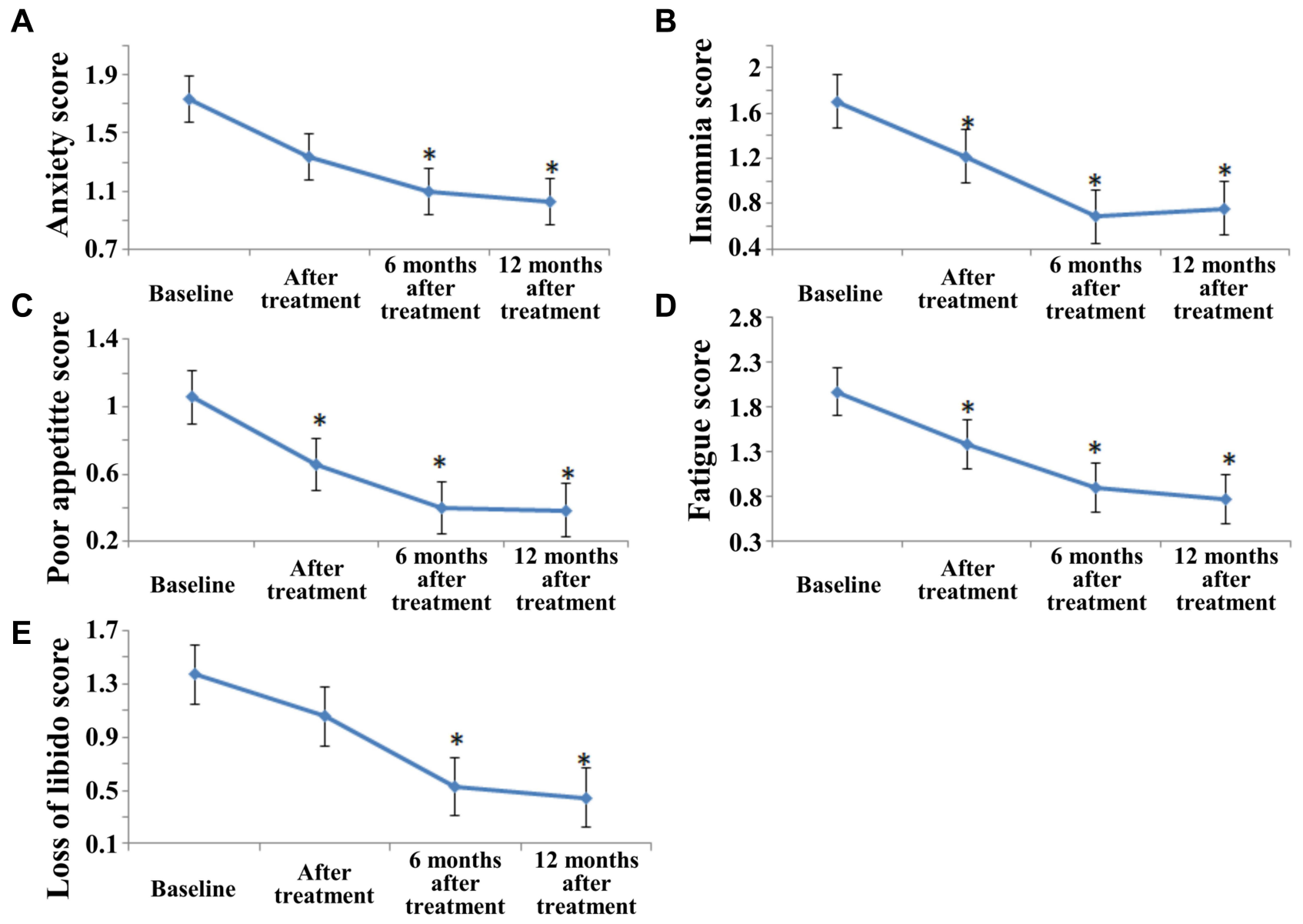

Figure 5 Scores of CMSS in related symptoms in the 3-course group, including anxiety score (A), insomnia score (B), poor appetite score (C), fatigue score (D) and loss of libido score (E). *Indicated a significant difference with a $p<0.05$.

disappear completely. However, after 6 months and 12 months, the physical and mental state of the patients were improved, which lead to the alleviation of anxiety and loss of libido.

The symptoms of abdominal pain were significantly improved before and after treatment in the 1-course group and the 3-course group. But the symptoms of dysmenorrhea, dyspareunia, lumbosacral pain and anal drop pain were not significantly improved after treatment in the 1-course group. Patients who were treated for 3 courses had significant improvements in pain, sexual pain, lower back pain and fall pain, suggesting that longer treatment course would possess certain benefits.

Due to the limited sample size, no longer-term observation was made, however, the possibility of long-term efficacy could not be ruled out. In terms of relieving abdominal pain symptoms and improving systemic symptoms, a continuous 3 months' treatment would be better.
The limitation of this study is that it is a retrospective analysis, lacking of randomized, doubleblind controlled trials, and hence therapeutic results cannot avoid the interference of patients' psychological factors. However, given the small sample size and the lack of evaluation of CMSS and SF36 in the 1-course group, some improvements may have still been observed. Therefore, more attention on these factors should be paid in future studies. Also, further studies are needed to objectively investigate the symptomatic treatment effect and quality of life with combined therapy of traditional Chinese medicine in the management of CPP.

\section{Conclusion}

Combined therapy of traditional Chinese medicine for three courses could relieve the pain and related symptoms of CPP, which helped improving patients' quality 

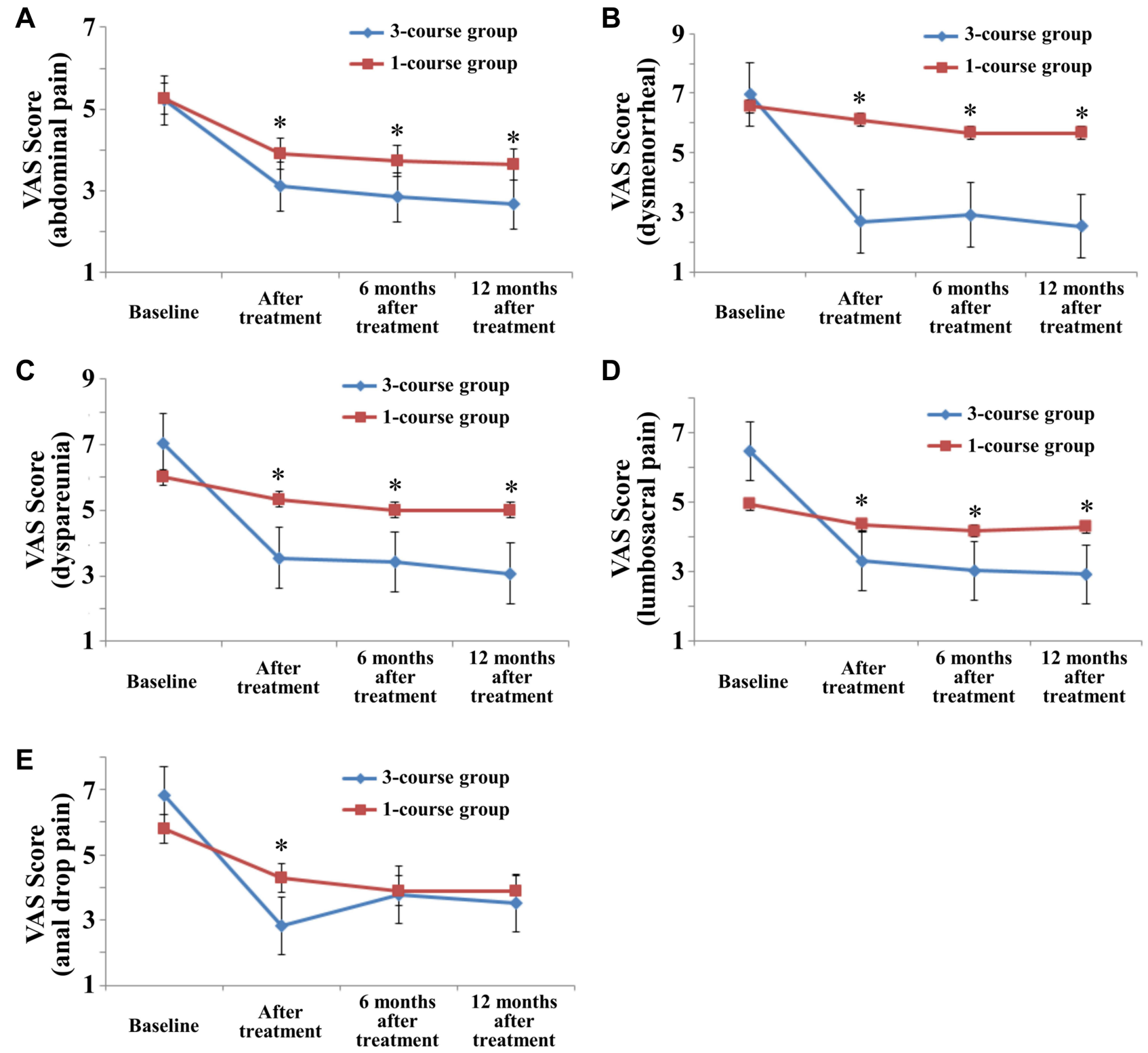

Figure 6 Comparisons of VAS scores, including abdominal pain (A), dysmenorrheal (B), dyspareunia (C), lumbosacral pain (D), and anal drop pain (E), in the I-course and the 3 -course group. *Indicated a significant difference with a $p<0.05$.

of life. Minor and reversible short-term side effects were observed in the current study. In the future, random double-blind trials study will be conducted to illustrate the efficacy of Combined therapy of traditional Chinese medicine on CPP.

Table 6 Adverse Events

\begin{tabular}{|l|l|}
\hline Symptom & Cases \\
\hline Mild skin rash & 16 \\
Oral ulcer & 1 \\
Irregular bleeding & 4 \\
Amenorrhea & 2 \\
Stomachache & 3 \\
\hline
\end{tabular}

\section{Acknowledgments}

This study was funded by 2019 Municipal Intangible Cultural Heritage Protection Special Fund Project (Chen's gynecological therapy) (Project No.: IX-13). We also want to extend our gratitude to Wen-jia Guo for his invaluable assistance.

\section{Disclosure}

The authors report no conflicts of interest in this work.

\section{References}

1. Yosef A, Allaire C, Williams C, et al. Multifactorial contributors to the severity of chronic pelvic pain in women. Am J Obstet Gynecol. 2016;215(6):760 e761-760 e714. doi:10.1016/j.ajog.2016.07.023 
2. Wang H, Liu S, Li J, et al. Investigation on prevalence and related factors of chronic pelvic pain in women in Changchun. China Pract Med. 2016;11:185-186.

3. Group EC, Branch OaG, Association CM. Guidelines for the diagnosis and treatment of endometriosis. Chin J Obstet Gynecol. 2015;31:161-169.

4. Association ICGoOaGBoCM. Diagnosis and treatment of pelvic inflammatory diseases. Chin J Obstet Gynecol. 2014;49:401-403.

5. Antibiotics Wgorogpfcao. Guidelines for Clinical Application of Antibiotics (2015 Edition). People's Medical Publishing House; July, 2015.

6. Hwang SK. Advances in the treatment of chronic pelvic pain: a multidisciplinary approach to treatment. Mo Med. 2017;114 (1):47-51.

7. Zhao S, Tang Y, Cai H, et al. Treatment of Danhong injection combined with Naoxintong capsule in acute coronary syndrome patients undergoing PCI operation: study for a Randomized Controlled and Double-Blind Trial. Evid Based Complement Alternat Med. 2018;2018:8485472.

8. Abd-El-Maeboud KH, Kortam MA, Ali MS, et al. A preliminary pilot randomized crossover study of uzara (Xysmalobium undulatum) versus ibuprofen in the treatment of primary dysmenorrhea. PLoS One. 2014;9(8):e104473. doi:10.1371/journal.pone.0104473

9. Chen YJ, Shimizu Bassi G, Yang YQ. Classic Chinese acupuncture versus different types of control groups for the treatment of chronic pain: review of Randomized Controlled Trials (2000-2018). Evid Based Complement Alternat Med. 2019;2019:6283912.
10. Hur MH, Lee MS, Seong KY, et al. Aromatherapy massage on the abdomen for alleviating menstrual pain in high school girls: a preliminary controlled clinical study. Evid Based Complement Alternat Med. 2012;2012:187163.

11. Stull DE, Wasiak R, Kreif N, et al. Validation of the SF-36 in patients with endometriosis. Qual Life Res. 2014;23(1):103-117. doi:10.1007/ s11136-013-0442-5

12. Xu D. Quantitative (Grade) Reference Standard for Simulated Interrogation Information. Jiangsu Science and Technology Press; 1997.

13. Xuejuan G, Zhifang Y, Shaoyin Y, et al. 40 cases of pelvic pain caused by cold coagulation and blood stasis treated by pelvic floor acupoint injection combined with warming meridians and dredging collaterals. Zhejiang J Trad Chin Med. 2017;12(3:2197. Chinese.

14. Yan-jun F, Ji-lan C, Jing-rui X, et al. Observation of the clinical therapeutic effects on chronic pelvic inflammation of interaction of damp and heat treated with the therapy for kidney notification and menstrual regulation and enema with Erteng decoction. World J Intergraded Trad West Med. 2017;12(3):399-402. Chinese.

15. Chang-hui L, Guang-hao C, Yan-ping W. Clinical study on the treatment of chronic pelvic pain (Damp-heat Stasis) sequelae of pelvic inflammatory diseases by collapse of Chinese medicine. Chin Foreign Med Treat. 2016;12:172-173.
Journal of Pain Research

\section{Publish your work in this journal}

The Journal of Pain Research is an international, peer reviewed, open access, online journal that welcomes laboratory and clinical findings in the fields of pain research and the prevention and management of pain. Original research, reviews, symposium reports, hypothesis formation and commentaries are all considered for publication. The manuscript

\section{Dovepress}

management system is completely online and includes a very quick and fair peer-review system, which is all easy to use. Visit http:// www.dovepress.com/testimonials.php to read real quotes from published authors. 